Harold A. McAlister and William I. Hartkopf (eds.)

\title{
The Gaseous Envelope Structure of the Eclipsing Binary System V448 Cyg $^{1}$
}

\author{
G. V. MANILOVA (VOLKOVA) \\ Astronomical observatory of Odessa State University, \\ T.G. Shevchenko Park, 270014 Odessa, Ukraine
}

\section{INTRODUCTION}

V448 Cyg (HD $\left.190967=\mathrm{BD}+34^{\circ} 3871\right)$ represents rather an uncommon case, where the primary component (indicated by stronger lines in the combined spectrum) is the star exhibiting mass loss. The system has a circumstellar envelope, formed by mass flow from a primary component filling its Roche lobe, and by a stellar wind that is stimulated by the duplicity of this system of two hot stars (B0 Ib + 09.5 V - see Glazunova et al. 1963). Ultraviolet, polarimetric, and spectral observations of V448 Cyg permitted us to form a model of the structure and parameters of this system's circumstellar envelope.

\section{OBSERVATIONS}

Polarimetric observations of V448 Cyg were performed by G.V. Volkova, in cooperation with N.M. Shakhovskoy, N.H. Minikulov, and A.I. Pikhun, from 1987 to 1989 , using the $1.25-\mathrm{m}$ mirror "AZT-11" telescope of the Crimean Observatory. These observations were made in 5 wide color bands that are close to the standard bands of the UBVRI system (Shakhovskoy et al. 1991). The data showed variability of the system's polarization parameters in all color bands. The amplitude of the polarization's variation degree $(\mathrm{P})$ in the $\mathrm{U}$ band is more than $0.4 \%\left(\sigma_{p}=0.05 \%\right)$, the amplitude of the position angle's variation $\theta$ more than $30^{\circ}\left(\sigma_{\theta}=4^{\circ} .4\right)$. In spite of the membership of V448 Cyg in the cluster NGC 6871, a determination of interstellar polarization by means of cluster stars is impossible because of a large dispersion in polarization values and directions for cluster members. Therefore a method was used for the calculation of interstellar polarization that a priori assumed the properties of interstellar and instrinsic polarization. In consequence, the interstellar component and a constant component of intrinsic polarization with a flat spectrum were distinguished in the observed polarization. Calculation of Fourier harmonics of the normalized Stokes parameters permitted us to distinguish the phase-variable part of the intrinsic polarization with spectral dependence typical of the intrinsic polarization of Be-stars. The constant component of intrinsic polarization fitted a probable extended disk-like optically-thin scattering envelope. The variable component fitted relatively dense condensations near the Lagrangian points. The mass of the extended envelope was estimated as $1.5 \times 10^{-8} \mathcal{M}_{\odot}$, the mass loss rate as $8.6 \times 10^{-7} \mathcal{M}_{\odot} /$ year, and the orbit's declination angle $i=82^{\circ} .4 \pm 5^{\circ} .5$. Vectors

${ }^{1}$ The author of this paper was unable to attend the Colloquium - eds. 


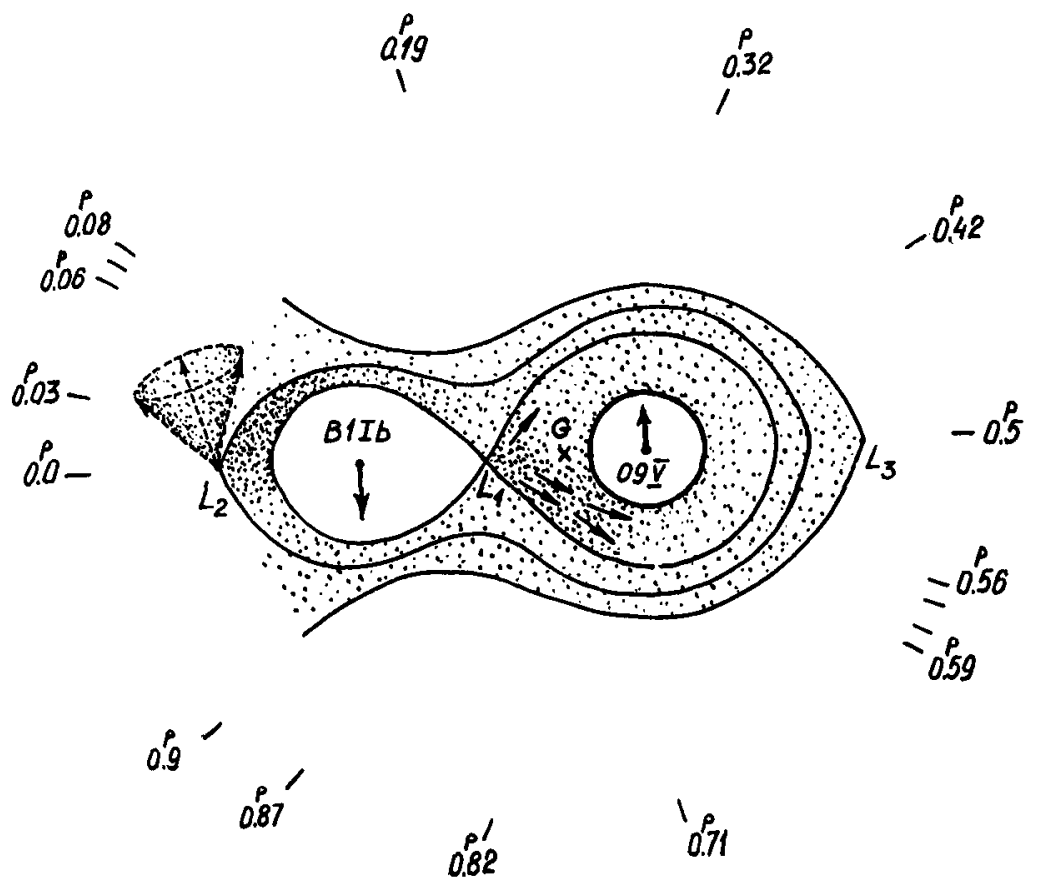

FIGURE 1. Model of the envelope structure of V448 Cyg

of V448 Cyg's intrinsic and interstellar polarization are parallel. This fact confirmed the hypothesis about correlation of the star-formation region's magnetic field direction and the position angle of intrinsic polarization of young stars.

Additional investigation of the V448 Cyg circumstellar envelope was performed by high-dispersion spectral data obtained in $1984-1989$ by G.V. Volkova, with the help of L.V. Glazunova and A.E. Tarasov, using the CCD-camera of the Crimean Observatory and the 6-m telescope of the Special Astrophysical Observatory (North Caucasus). Fifteen spectra with $\mathrm{D}=3,6$, and $14 \AA / \mathrm{m}$ were obtained at all phases of the orbital period. The obtained profile of the $\mathrm{H} \alpha$ line in the spectrum of this star strongly varies with the phase of orbital period: the profile has two emission peaks ( $\sim 0.15$ over the continuum), P Cyg-like emission, inverted P Cyg-like emission, and clean absorption. It is shown that the main part of these changes correlates with the phase of orbital period. The change in the $\mathrm{H} \alpha$-profile emission's residual, that was determined by subtraction of the binary's combined photosphere profile from the observed profile, was studied. Change in radial velocity of the short-wave (V) and long-wave (R) emission components occurs in phase with the $B$-star radial velocity curve. The region where these components were forming was found near the $L_{2}$-point between the first and the second Roche surfaces (Figure 1). The change in radial velocity of the central, non-shifted emission component occurs in phase with the radial velocity 
curve of the more massive 0 -star, which means that this emission component's formation region lies inside the O-star's Roche lobe (Volkova et al. 1992).

\section{CONCLUSION}

Polarimetric and spectral data have shown that the V448 Cyg circumstellar envelope has a disk-like structure with its most dense condensation near the second Lagrangian point.

\section{REFERENCES}

Glasunova, L.V., Karetnikov, V.G., \& Jutsenko S. 1963, Sov. Astron. J., 63

Shakhovskoy, N.M., Volkova, G.V., Manilov, Yu.L., \& Minikulov, N.Kh. 1991, Crimean Journal of Astrophysics, 2, in press.

Volkova, G.V., Glazunova, L.V., \& Tarasov, A.E. 1992, Sov. Astron. J., in press. 